\title{
The Innovation of Batch Adjusting Account after Accounting Based on BSS and CBSS
}

\author{
Meng-lin Liu ${ }^{1}$, jian-guang Liu ${ }^{2}$ \\ ${ }^{1}$ College of Information Science and Engineering Zao Zhuang University Zao Zhuang, \\ shandong, 277100, China; \\ ${ }^{2}$ Zao Zhuang Unicom Company, Zao Zhuang, shandong, 277100, China .
}

Keywords: Innovation, Adjusting Account, Batch Account.

\begin{abstract}
The adjustment of batch accounts is going to start after the account is completed. The adjustment of batch accounts is one of the important links of accounting processing in the Information Service. The timeliness and accuracy of the adjustment of batch accounting is directly related to the success or failure of accounting treatment, and it is related to the responsiveness of market demand and the credibility of the market. On the basis of BSS and CBSS, this paper has carried on the innovation in order to accomplish the above mission. By applying this innovation, the work has been reduced in difficulty, the working hours are saved, and the procedural level of billing processing has improved. Thus opened up a new thinking about batch adjusting account after accounting.
\end{abstract}

\section{Introduction}

Since August 2016, the agent's calls (including BSS and CBSS) have been changed from batch giving away phone bills to batch adjusting account after the account has completed. But the adjustment of batch accounts after the accounts need to fully and correctly solve the following problems:

Firstly: Relieving the great difference between the reimbursement documents and warehousing documents. Although the reimbursement process has given the necessary items about the adjustment of batch accounting, for example : The account number, and the remaining amount, reduce the total amount of the total arrears, etc. But it does not contain detailed account items and the corresponding arrears data. While BSS and CBSS's batch adjusting account after accounting, especially CBSS, must have the details of the accounts and the corresponding arrears data, so there is a great difference. The handle of batch account adjustment need to eliminate these differences. The way to eliminate these differences is changing the total amount of adjustment of a single number into a single number containing detailed accounts, the amount of detail.

Secondly: Because the approval process takes a certain amount of time, telephone fare may have changed in the process of adjusting account, so it must exclude these changes, Out of account number does not meet the provisions of the account number.

Thirdly: There are two databases are involved which are BSS and CBSS in the process of adjusting accounts. So it needs to be done separately on the two databases.

Fourthly: Excluding the duplicate reimbursement number in the same period of reimbursement documents , and appropriate treatment.

Fifthly: The audit of batch adjusting account after accounting must have the appropriate auditing procedures to ensure the accuracy of the batch adjustment after the accounting.

Sixthly: The accounts processing of batch adjusting account after accounting has requirements about the time limited, so it must be properly and timely processing within the specified time.

In order to solve the practical problems listed above, I make innovations for the batch adjusting account after accounting On the basis of full investigation. It is that remove duplicate reimbursement number, detailed data of current need to reextract the arrears after the transfer accounts (including account number, contract number, user identification, payment term, net cost, current cost, and 
arrears, etc.), the extraction have not eliminated the user reimbursement reimbursement fee, will be removed from the amount of the remaining amount the details of arrears extracted according to the illumination of the fine, do not meet the specified user account, excluding arrears from the extraction of the detailed record, so as to meet the demand of storage for the batch documentation, respectively to produce BSS and CBSS batch account reconciliations in the audit file, storage and execution, and then use the program audit, correct test batch account reconciliations, thus successfully completed batch account reconciliations.

\section{The highlights of this innovation}

The focus and difficulty of this innovation is that reimbursement documents with the total adjustment amount of the account after adjustment can be transformed into the batch documents witch can meet warehousing requirements, and eliminate the amount of the remaining amount, the user does not owe arrears, and does not meet the requirements of the transfer number and audit. Then The adjustment of batch accounts after accounting is successfully realized .

\section{The realization process of this innovation}

\section{The first: Create related tables in the database, and extract arrears details on BSS}

One table is used for summary, backup the original account data .Account these data, the account after adjustment will be finished. The structure of this table is following:

zz_t_zhtz_bz(sn varchar2(30), syje number(10,2),

cycle_id varchar2(8), fl_no varchar2(10),

remark varchar2(100), user_id number(30),

net_type_code varchar2(4),

input_date date default sysdate , tjje number(12,2), dyje number(12,2));

The second table is used to get the account period from table ZZ_T_ZHTZ_BZ.The structure of this table is following:

zz_t_zhtz_201608

as select trim(sn) sn ,tjje,cycle_id,fl_no,remark,net_type_code,user_id

from zz_t_zhtz_bz

where cycle_id $=* * * *$;

The third table is used to store the arrears data for broadband, 2G, 3G and 4G. The structure of this table is following:

zz_t_qf_zhtz_201604_mid as --bss

select a1.serial_number,

a1.acct_id, a1.user_id,

a1.net_type_code,

a1.cycle_id,

a1.integrate_item_code,

b1.item_name ,sum(nvl(a1.fee,0))/100.00 yjfy,

sum(nvl(a1.balance,0))/100.00 fee

from ucr_act2.ts_b_bill@siact2db a1,

ucr_param.td_b_item@siact2db b1 ,

zz_t_zhtz_201608 a

where a1.user_id=a.user_id

and a1.integrate_item_code $=$ b1.item_id $(+)$

and a1. cycle_id=****

and a1.eparchy_code='0632'

group by a1.serial_number, a1.acct_id,

a1.user_id, a1.net_type_code, a1.cycle_id ,

a1.integrate_item_code, b1.item_name having sum(nvl(a1.balance,0)) >0 
order by a1.serial_number,a1.cycle_id ;

\section{The second: Import the data whitch required adjusting}

The data after approval of non_investment process is summarized and is imported into the table zz_t_zhtz_bz.

\section{The third: Remove duplicate reimbursement}

Through the following SQL statement,the duplicate reimbursement is removed.

select trim(sn),count $\left(^{*}\right)$

from zz_t_zhtz_201608

where cycle_id $=* * * *$

group by trim(sn) having count $(*)>1$

The fourth: Obtain the billing mark USER_ID and the net_type_code of the billing number

The specific processes for obtaining bill_marking and networking are as follows:

begin

update zz_t_zhtz_201608

a --bss user_id, net_type_code

set a.user_id=(select b.user_id

from ucr_crm2.tf_f_user@sicrm2db b

where b.eparchy_code='0632'

and trim(a.sn)=b.serial_number

and b.remove_tag $<=1)$,

a.net_type_code=(select b.net_type_code

from ucr_crm2.tf_f_user@sicrm2db b

where b.eparchy_code='0632'

and trim(a.sn)=b.serial_number and b.remove_tag $<=1$ );

commit;

update zz_t_zhtz_201608

a --cbss user_id、net_type_code

set a.user_id=(select b.user_id from un_like.tf_f_user@lscx1 b

where b.eparchy_code='0632' and trim(a.sn)=b.serial_number and b.remove_tag $<=1$ ),

a.net_type_code=(select b.net_type_code from un_like.tf_f_user@lscx1 b

where b.eparchy_code='0632' and trim(a.sn)=b.serial_number and b.remove_tag $<=1$ )

where a.user_id is null and a.net_type_code is null;

commit;

end;

\section{The Fifth: Extract details}

First we can get in the file marked billing reimbursement by the follow code:

select distinct user_id ||',' from zz_t_zhtz_201608

where net_type_code='50';

And then receive arrears details, the specific statements for:

select

serial_number,acct_id,user_id,net_type_code,cycle_id,integrate_item_code, sum( balance)/100.00

fee from ucr_act6.ts_b_bill b

where b.balance $>0$

and b.eparchy_code='0632'

and b.cycle_id $=* * * *$

and b.user_id in()

group by serial_number,acct_id,user_id, net_type_code, cycle_id,integrate_item_code ;

Finally, the extracted data is imported into the default data table zz_t_qf_zhtz_201604_mid. 


\section{The Sixth: Delete the data without arrears}

The specific processes for deleting the data without arrears are as follows:

delete from zz_t_zhtz_201608

where trim(sn) not in (

select distinct serial_number from zz_t_qf_zhtz_201604_mid);

\section{The Seventh: According to the amount of reimbursement arrears and user details, readjust the storage details}

Core program for:

declare

type fee_max_r is record (sn varchar2(30),tjje number(10,2));

fee_maxr fee_max_r;

integrate_item_code1 zz_t_sy.integrate_item_code\%type;

i_fee number $(10,2)$ default 0 ;

i_y number $(10,2)$ default 0 ;

begin

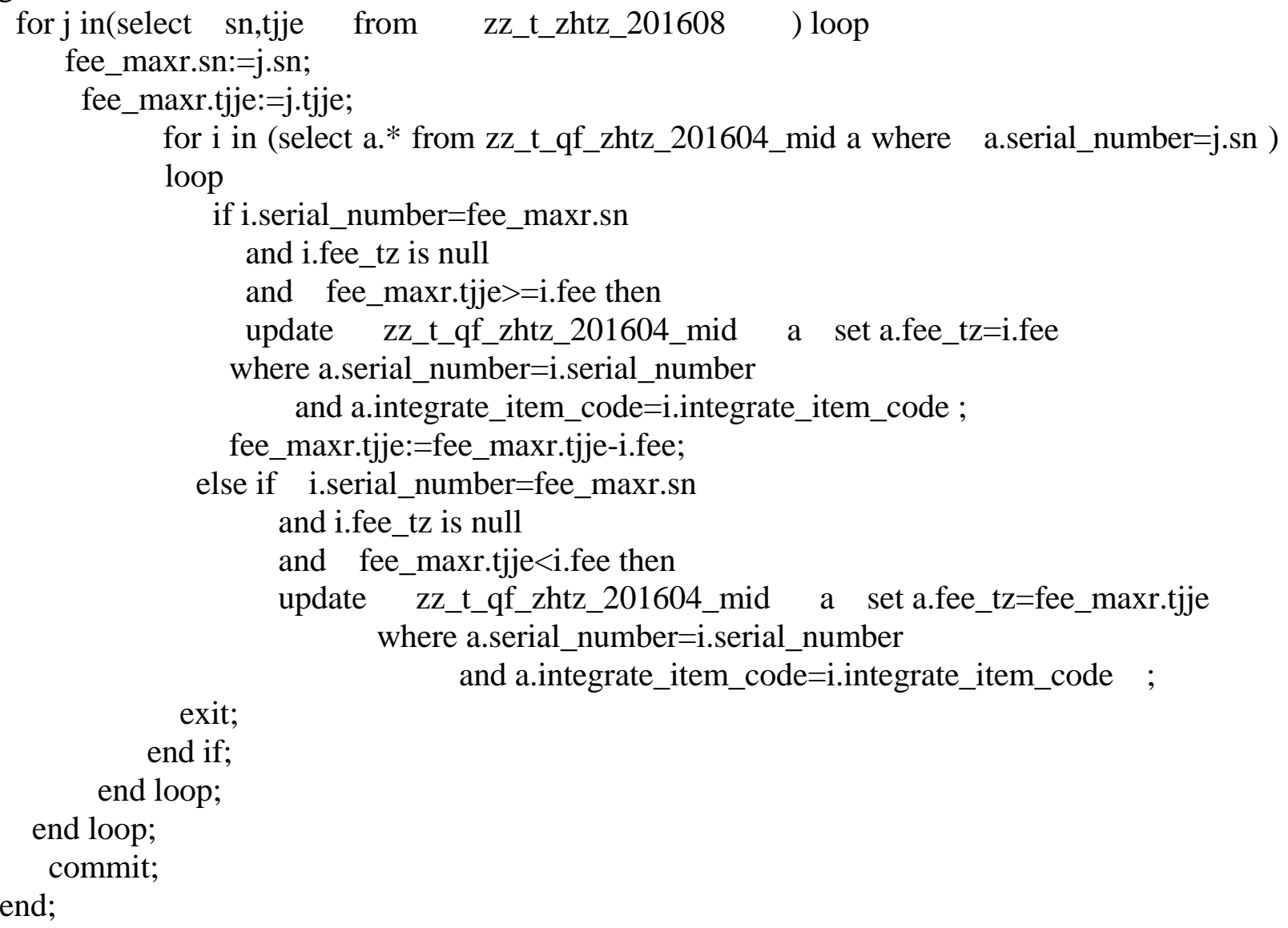

\section{The Eighth: Extract the storage file of2G,3G and 4G}

Core program for:

select a1.serial_number,a1.net_type_code,a1.integrate_item_code,a1.fee_tz fee,a1.cycle_id

from zz_t_qf_zhtz_201604_mid a1

where a1. net_type_code $=50$

and a1.fee_tz $>0$

and a1.integrate_item_code $<>1000$

and a1.integrate_item_code $<>10074$

and serial_number not in ('***********'); 
The Ninth: Import the generated documents respectively in the BSS and CBSS interface, so as to complete the batch adjusting account after accounting

The specific audit procedures are as follows:

select batch_id,

(case when adjust_type='0' then ' Compensation fee '

when adjust_type='1' then 'Refund '

end ) adjust_type,

( case when adjust_mode $=$ '0' then ' By amount '

when adjust_mode='1' then ' In proportion '

end ) adjust_mode,

( case when act_tag $=\quad$ '0' then ' Import status '

when act_tag='1' then ' Association state '

when act_tag='2' then ' Auditing;'

when act_tag='3' then ' Audit not through '

when act_tag $=$ ' 4 ' then ' Can perform '

when act_tag='5' then ' End of processing '

when act_tag='6' then ' Void / buyback '

end ) act_tag,

(case when recv_tag=' 0 ' then ' Adjust excess amount to void '

when recv_tag='1' then ' Adjust the excess amount to the grant '

end ) recv_tag,

count(*),

sum(adjust_fee) $/ 100.00$,

sum(nvl(real_fee,0))/ 100.00

from ucr_act2.tf_b_adjustalog_chk@siact2db

where batch_id in $(* * * *)$

group by batch_id, adjust_type, adjust_mode,act_tag, recv_tag;

\section{The significance of this innovation}

By applying this innovation, the work has been reduced in difficulty,the working hours are saved, and the procedural level of billing processing has improved. Thus opened up a new thinking about batch adjusting account after accounting .

\section{Conclusion}

From August 2016 to May 2017,after nearly a year of use,practice has proved that the innovation provides a more efficient, more accurate and more efficient services. The innovation has some practical value.

\section{References}

[1] P. D.Bo: MySQL technology insider(Higher Education Press,China 2015).

[2] J.H. Feng, G.L and Li, J.Y.Li:IEEE Transactions on Knowledge and Data Engineering, Vol.213( 2011) No.10,P.223-227.

[3] G.L. Li, J.H. Feng, X.F. Zhou and J.Y. Wang: The VLDB Journal - The International Journal on Very Large Data Bases ,Vol.23( 2011) No.3,P.23-25.

[4] P.He and Y.Liu: Electronic Technology \& Software Engineering, Vol.89(2014) No.5, p. 42-43.( In Chinese) 